\title{
Serum Paraoxonase 1 Activity and Oxidative Stress in Pediatric Patients with Pulmonary Tuberculosis
}

\author{
Emel Torun ${ }^{\mathrm{a}}$ Ahmet Hakan Gedik ${ }^{\mathrm{a}}$ Erkan Cakir ${ }^{\mathrm{b}}$ Tarik Umutoglu ${ }^{\mathrm{c}}$ \\ Ozlem Gok ${ }^{d}$ Ulkan Kilic ${ }^{d}$ \\ Departments of a Pediatrics, ${ }^{b}$ Pediatric Pulmonology, ${ }^{\mathrm{c} A n e s t e s i o l o g y}$ and d Medical Biology, Medical Faculty, \\ Bezmialem Vakif University, Istanbul, Turkey
}

\section{Key Words}

Tuberculosis · Oxidative stress · Total oxidant status .

Paraoxonase 1

\begin{abstract}
Objectives: The aim of this study was to determine the oxidative stress and paraoxonase 1 (PON1) levels in children with pulmonary tuberculosis (TB) compared to healthy controls, and to examine the association of demographical with oxidative stress. Subjects and Methods: Forty children diagnosed with pulmonary TB and 40 age- and gender-matched healthy controls were enrolled in the study. Serum total antioxidant status (TAS), total oxidant status (TOS) and PON1 levels were measured. The oxidative stress index (OSI) was calculated to indicate the degree of oxidative stress. Results: The TAS levels were lower ( $1.73 \pm 0.5$ vs. $2.54 \pm 1.2 \mu$ mol Trolox Eq/l) while TOS levels were significantly higher (26.9 \pm 14.4 vs. $\left.13.4 \pm 7.7 \mu \mathrm{mol} \mathrm{H}_{2} \mathrm{O}_{2} \mathrm{Eq} / \mathrm{l}\right)$ in the TB group than in the controls $(p<0.001)$. The OSI was significantly higher in the TB group than in the controls $(21.2 \pm 5.1$ vs. $6.5 \pm 4.9$ units, $p=0.006)$. Serum PON1 levels were significantly lower in the TB group than in the controls (14.2 \pm 13.2 vs. $28.4 \pm 17.3 \mathrm{U} / \mathrm{l}$, $p<0.001)$. The lower PON1 levels correlated with TAS and OSI levels but not with anthropometric parameters $(r=$
\end{abstract}

$0.264, p=0.018$ and $r=-0.255, p=0.023$, respectively). Conclusion: The TOS and OSI levels were higher and the TAS and PON1 levels were lower in pediatric patients with pulmonary TB when compared to healthy controls. This indicates greater oxidative stress in the patients.

(c) 2014 S. Karger AG, Basel

\section{Introduction}

Free radical formation, which is a result of tissue metabolism, has the potential to cause organ damage. The detrimental effects of free radicals are minimized by the antioxidant capacity and repair mechanisms of the cells. Oxidative stress is the term used for an imbalance between prooxidants and antioxidants; it can lead to irreversible changes at the cellular level [1]. Disturbances in the equilibrium of the oxidant and antioxidant systems are a result of the rapid production of reactive oxygen species (ROS) that occur during infection, trauma, exposure to pollutants, the intake of drugs or unusual food and the administration of oxygen. Free radical burden has been reported as an important factor in the pathogenesis of childhood diseases such as bronchiolitis, asthma, congenital heart disease and hypertension [2-5]. The role of 
oxidative stress in the pathogenesis of childhood tuberculosis (TB) has not been defined to date.

Pulmonary TB is still a major health problem in Turkey and, not surprisingly, infants and children are more susceptible than adults to diseases related to Mycobacterium tuberculosis (MTB) [6]. The innate and adaptive immune response of the host to MTB determines clinical outcome in the very early phase of the infection. However, immune responses to MTB and the pathogenesis of TB disease are both complex and not completely understood. MTB is an intracellular pathogen, which, after being inhaled by the host, is phagocytosed in the lungs by antigen-presenting cells such as alveolar macrophages, lung parenchyma macrophages and dendritic cells. These cells go on to elicit local inflammatory responses [6]; this leads to a respiratory burst resulting in the production of large amounts of ROS and reactive nitrogen intermediates for the purpose of destroying ingested microorganisms [7]. Although the enhanced level of free radical production is designed to contribute to the host's defense against microorganisms, it may inadvertently damage the host [8]. There is a report that links pulmonary TB in adults with inflammation-related oxidative stress involved in the pathogenesis of dysfunction [9]. The oxidative burst in human phagocytes, especially with monocytes and macrophages, contributes to the killing of mycobacteria as a part of innate immunity $[10,11]$. There are not yet any studies about the oxidative stress in childhood TB.

Paraoxonase 1 (PON1) is an antioxidizing enzyme that contributes to the hydrolysis of lipid peroxides into oxidized lipoproteins, and it has been associated with diseases characterized by high oxidative stress such as cardiovascular disease and diabetes [12]. Oxidative stress downregulates serum PON1 expression due to the changes in the redox status [13]. There is no existing report on PON1 activity in pediatric pulmonary TB. In this study, we investigated the oxidative status and PON1 activity in pediatric TB compared to in healthy age- and gendermatched children.

\section{Subjects and Methods}

This cross-sectional study included 40 children who were admitted to the Department of Pediatric Pulmonology with a diagnosis of pulmonary TB. The control group consisted of 40 age- and gender-matched children who had been admitted at the same time to our outpatient clinic for reasons other than infectious and inflammatory diseases. This study was approved by the Institutional Ethics Committee. Written informed consent was obtained from the parents of the study and control groups. The study was conducted between January 2012 and May 2013. TB diagnosis was based on respiratory symptoms, radiological findings, positive tuberculin skin tests, a history of contact with someone with active $\mathrm{TB}$, acid-fast bacillus and positive MTB cultures. All the subjects met the criteria for a strong suspicion of TB.

The patients were examined by both a pediatrician and a pediatric pulmonologist and were evaluated according to a protocol which included taking down their history and performing a physical examination. Their history concerned household contacts and comorbidities such as malnutrition, chronic liver or kidney disease, immune suppression from drugs or disease and diabetes. No patients in either group had a history of immunosuppressive drug use, immune deficiency or chronic disease. Malnutrition was evaluated by body mass index standard deviation score (BMI SDS) and relative weight. Standing height was measured to the nearest 0.1 $\mathrm{cm}$ with a Harpenden fixed stadiometer. Body weight was measured on a SECA balance scale to the nearest $0.1 \mathrm{~kg}$, with each subject dressed in a light T-shirt and shorts. Relative weight was calculated as weight/midpoint of the weight of people of the same height $\times 100$.

The standard biochemical parameters including albumin, total protein and complete blood count were measured for the TB and control groups. Albumin and total protein were measured with the colorimetric technique (ROCHE Cobas 8000 , USA), and complete blood count was measured with the impedance technique (Sysmex XT 1800I, USA).

The total antioxidant status (TAS) was measured using a novel automated direct measurement method for TAS developed by Erel [14] (Rel Assay Diagnostics ${ }^{\circledR}$, Gaziantep, Turkey). With this method, antioxidants in the sample reduced dark blue-green-colored 2,2'-azino-bis(3-ethylbenzothiazoline-6-sulfonic acid) radicals to a colorless reduced form. The change of absorbance at $660 \mathrm{~nm}$ is related to the total antioxidant level of the sample. This method determines the antioxidative effect of the sample against the potent free radical reactions initiated by the produced hydroxyl radical. The results are expressed as the micromolar Trolox equivalent per liter.

The total oxidant status (TOS) of serum was measured using an automated colorimetric measurement method developed by Erel [15] (Rel Assay Diagnostics). Again, briefly, oxidants present in the sample oxidize the ferrous ion-chelator complex to ferric ion which makes a colored complex with chromogen $S$ in an acidic medium. The color intensity, which can be measured spectrophotometrically, is related to the total amount of oxidant molecules present in the sample. The results are expressed as the micromolar hydrogen peroxide equivalent per liter.

Oxidative stress index (OSI) is the percentage ratio of the level of TOS/TAS. The resulting micromolar unit of TAS is converted to millimoles per liter, and the OSI value is calculated according to the following formula: OSI $=\operatorname{TOS}\left(\mu \mathrm{mol} \mathrm{H}_{2} \mathrm{O}_{2} \mathrm{Eq} / \mathrm{l}\right) / \mathrm{TAS}(\mu \mathrm{mol}$ Trolox Eq/l).

The PON1 enzyme activity was measured by using commercially available kits (Rel Assay Diagnostics). The fully automated PON1 activity measurement method consists of two different sequential reagents. The first is an appropriate Tris buffer, containing calcium ion which is a cofactor of the PON1 enzyme. The second is paraoxon ethyl that hydrolyses paraoxon and increases the adsorbance at $412 \mathrm{~nm}$. The linear increase of the absorbance of $p$-nitrophenol produced from paraoxon is subtracted from the to- 
tal rate of hydrolysis. The molar absorptivity of $p$-nitrophenol is $18,290 \mathrm{M}^{-1} \mathrm{~cm}^{-1}$ and 1 unit of paraoxonase activity is equal to $1 \mathrm{~mol}$ of paraoxon hydrolyzed per liter per minute at $37^{\circ} \mathrm{C}[16]$.

\section{Statistical Analysis}

Statistical analysis was performed with the Predictive Analytics software statistics program, version 13.0. Median and interquartile ranges were used for metric variables. The Mann-Whitney U test was used to calculate the difference of two parameters in groups, and the Kruskal-Wallis test was used for calculating the difference of two parameters in groups with more than two in the same group and between different groups. Multiple comparisons were made using the Pearson correlation. Linear regression analysis was used to evaluate the effects of factors on dependent variables. Categorical data were evaluated using the $\chi^{2}$ test; $p<0.05$ was accepted as statistically significant.

\section{Results}

In the TB group, 27 (67.5\%) children had a history of household contact and $33(82 \%)$ had positive tuberculin skin tests. All the patients had radiological findings consistent with $\mathrm{TB}$ nonresponsive to nonspecific therapy with antibiotics, and 17 (42\%) had positive MTB cultures. The dominant clinical features of the children with TB were cough in 32 (80\%), fever in 19 (47.5\%), weight loss in 18 (45\%), night sweats in $17(42.5 \%)$ and weakness and anorexia in 20 (50\%). They all responded to anti-TB therapy.

The BMI SDS and relative weight were lower in the TB group than in the healthy controls, but did not reach a statistically significant level $(\mathrm{p}=0.33$ and 0.81 , respectively; table 1).

The laboratory findings of both the study and control groups revealed no statistically significant difference between the biochemical parameters. Mean hemoglobin $(12.1 \pm 1.1$ and $12.9 \pm 1.3 \mathrm{~g} / \mathrm{dl})$, albumin $(4.2 \pm 0.4$ and 4.5 $\pm 0.2 \mathrm{~g} / \mathrm{dl})$ and total protein $(6.9 \pm 0.6$ and $7.1 \pm 0.2 \mathrm{~g} / \mathrm{dl})$ were not statistically different between the two groups ( $\mathrm{p}=1.13,0.74$ and 0.46 , respectively).

The TAS levels were lower and the TOS levels were significantly higher in the TB group than in the control group $(\mathrm{p}<0.001)$. The OSI was significantly higher in the TB group than in the controls $(\mathrm{p}=0.006)$. Serum PON1 levels were significantly lower in the TB group than in the controls ( $\mathrm{p}<0.001$; fig. 1$)$. There was a significant positive correlation between the PON1 levels and the OSI $(\mathrm{r}=$ $-0.255, \mathrm{p}=0.023)$ and TAS levels $(\mathrm{r}=0,264, \mathrm{p}=0.018$; table 2). The Pearson correlation analysis disclosed that levels of TAS, TOS, OSI and PON1 did not correlate with anthropometric parameters (table 2).
Table 1. Characteristics of children in the TB and control groups

\begin{tabular}{lccl}
\hline & $\begin{array}{l}\text { Children with TB } \\
(\mathrm{n}=40)\end{array}$ & $\begin{array}{l}\text { Healthy controls } \\
(\mathrm{n}=40)\end{array}$ & $\mathrm{p}$ \\
\hline Age, years & $9.2 \pm 4.7$ & $8.3 \pm 4.2$ & 0.97 \\
Male/female, $\mathrm{n}$ & $18 / 22$ & $20 / 20$ & 0.5 \\
BMI SDS & $-0.62 \pm 1.2$ & $-0.3 \pm 0.8$ & 0.33 \\
Relative weight & $98.2 \pm 13.1$ & $102.2 \pm 11.5$ & 0.81 \\
\hline
\end{tabular}

Values represent mean \pm SD unless otherwise indicated.

\section{Discussion}

This study showed that oxidative stress levels were higher and antioxidant status was lower in the children with TB than in the healthy controls. Normally, ROS are produced in the cells at basal levels as a result of the metabolic process, but they are contained and neutralized by various antioxidant systems that exist in every cell. The high levels of oxidative stress in TB patients could be due to the increased production of ROS brought about by a major response of macrophages to inflammatory stimuli or infection, that occur in the initial phase of TB infection. This response of the macrophages disrupts the balance between the oxidant and antioxidant systems in favor of oxidative damaging [17]. Our findings confirmed the contribution of oxidative stress to the pathogenesis of active TB [18-20]. Dalvi et al. [18] reported that oxidative parameter levels were significantly increased in adult subjects who had pulmonary and extrapulmonary TB. They also reported that the increased oxidative stress was not completely reversed after 6 months of anti-TB treatment. Studies using serum malondialdehyde concentration for a measure of lipid peroxidation also revealed significantly higher oxidative stress in TB patients than in healthy controls $[19,20]$. However, we did not measure a selected antioxidant parameter but rather the total antioxidant and oxidant status that necessarily reflect the actual free radical burden in these patients.

The antioxidant homeostasis depends on multiple factors such as antioxidant enzymes, micronutrients and dietary composition. Dietary antioxidant vitamin status is usually poor in TB patients of lower socioeconomic status and this could possibly explain the low levels of antioxidant potential of the TB patients in our study. Malnutrition might be one of the factors that reduced the antioxidant response. Although the lower BMI SDS and relative weight in the TB group did not reach statistically significant levels compared to those in the healthy controls, it 
Table 2. Pearson correlation analyses of TAS, TOS, OSI and paraoxonase levels in the TB group

\begin{tabular}{|c|c|c|c|c|c|c|c|c|}
\hline & \multicolumn{2}{|l|}{ TAS } & \multicolumn{2}{|l|}{ TOS } & \multicolumn{2}{|l|}{ OSI } & \multicolumn{2}{|l|}{ PON1 } \\
\hline & $\mathrm{r}$ & $\mathrm{p}$ & $\mathrm{r}$ & $\mathrm{p}$ & $\mathrm{r}$ & $\mathrm{p}$ & $\mathrm{r}$ & $\mathrm{p}$ \\
\hline Age (years) & 0.161 & 0.155 & -0.037 & 0.746 & -0.141 & 0.211 & 0.054 & 0.635 \\
\hline Gender & -0.169 & 0.134 & 0.366 & 0.001 & 0.249 & 0.026 & -0.298 & 0.007 \\
\hline BMI SDS & 0.077 & 0.554 & -0.116 & 0.371 & $-0,031$ & 0.811 & 0.085 & 0.509 \\
\hline Relative weight & 0.077 & 0.741 & -0.264 & 0.247 & -0.193 & 0.402 & -0.131 & 0.572 \\
\hline PON1 (U/l) & 0.264 & 0.018 & -0.179 & 0.112 & -0.255 & 0.023 & - & - \\
\hline
\end{tabular}

Fig. 1. Levels of serum TAS, TOS, OSI and $\mathrm{PON} 1$ in the TB and control groups.

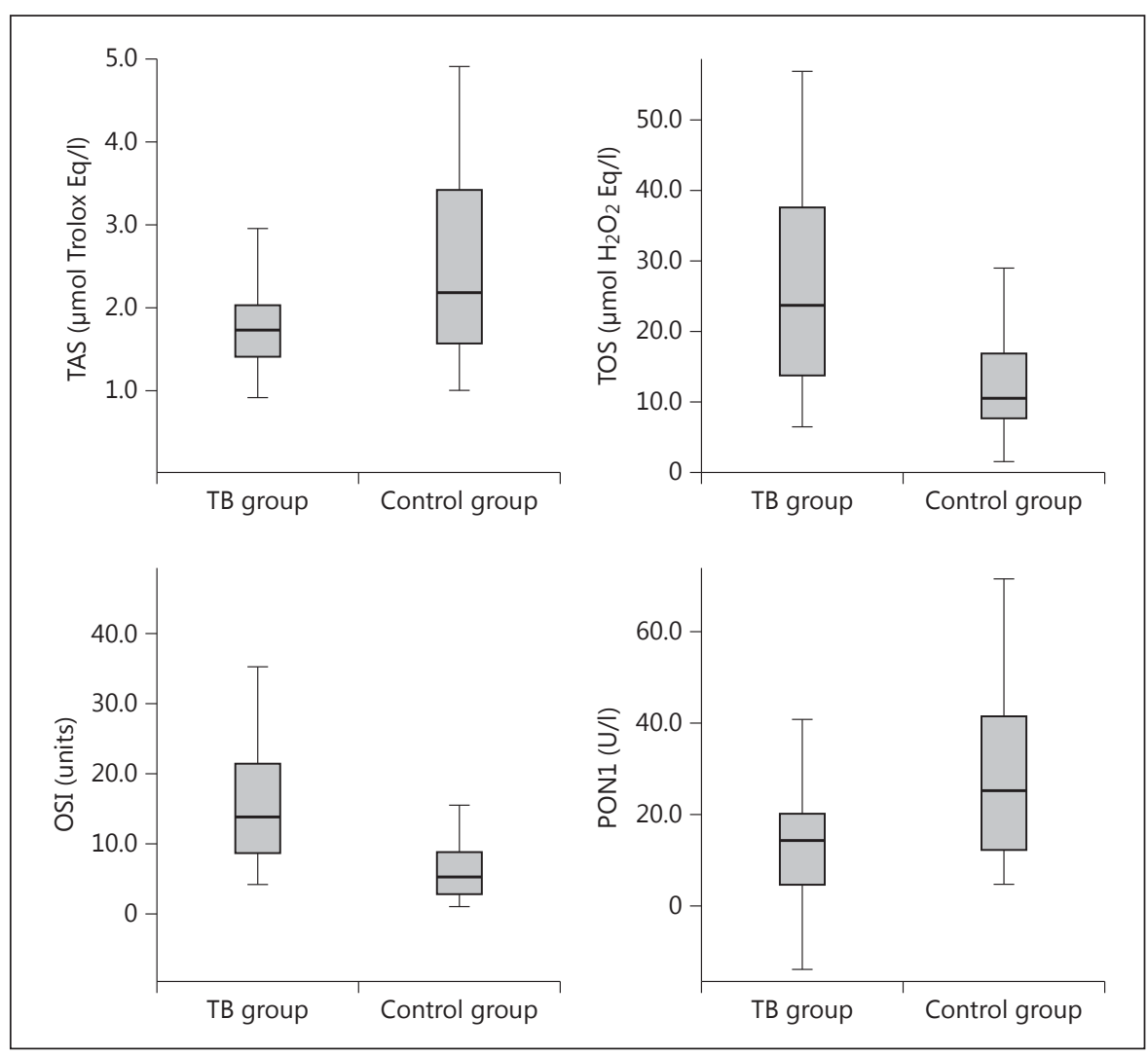

could be assumed that children with TB were mildly malnourished due to an acute starvation stage that was not yet reflected in the anthropometric parameters in the initial phase of the infection.

Our results for pediatric patients are similar to those in studies on adults $[21,22]$. One study, investigating the antioxidant status of healthy and TB-infected individuals from a community with a high incidence of TB as well as in TB patients at various stages of anti-TB drug treatment, showed that the active TB patients had significantly lower levels of TAS than the healthy controls [22]. In addition, the total antioxidant status of TB patients correlated with antioxidant vitamin levels. Similar results were revealed in the study by Sharda [1] on the role of free radicals in childhood TB. Antioxidant levels were found to be lower in the TB cases (18 of the respiratory system, 6 of the lymph nodes and 5 of the central nervous system) than in the healthy controls, with the lowest levels of antioxidant vitamins being observed in the central nervous system TB when compared to the TB of the respiratory system and lymph nodes. 
PON1 is a calcium-dependent esterase that hydrolyzes a broad spectrum of oxidized lipids [23]. Owing to an increase in lipid and protein oxidation products and a decrease in antioxidant enzymes and vitamins, oxidative stress is associated with decreased PON1 expression and activities [24]. Serum PON1 expression is downregulated by oxidative stress [25]. In our study, serum PON1 activity was significantly lower in the pulmonary TB patients than in the controls, indicating that the OSI was negatively correlated with PON1 levels and that antioxidant levels were positively correlated with PON1 levels in the TB patients. The high OSI and low TAS levels could be related to low PON1 activity which led to increased susceptibility to lipid peroxidation.

The mechanism of the low PON1 activity in TB patients is not well known. Proinflammatory cytokines play a major role in the local immunity against MTB [26]. These peptides contribute to antimicrobial chemokines and elicit a local inflammatory response. Studies have shown that proinflammatory mediators decrease PON1 activity and its gene expression $[27,28]$; they show a reduction in PON1 mRNA expression in vitro by IL-6, IL-1 and TNF- $\alpha$. It might be speculated that the low level of paraoxonase activity in TB patients was associated with the proinflammatory cytokines that are competent in modulating PON1 expression. Our findings confirmed those of previous studies regarding the level of total antioxidant status and paraoxonase activity in TB in adult patients. Selek et al. [29] report that total oxidant status was enhanced while serum basal and salt-stimulated PON1 and arylesterase activities were lower in TB patients than in controls. Naderi et al. [23] also report that the serum PON1 activity was lowest in adult TB patients when compared to non-TB pulmonary disease patients and healthy controls.

\section{Conclusion}

The OSI was high and PON1 levels were low in our pediatric patients with pulmonary TB. These findings further support the general idea that mycobacterial infections could induce ROS which might promote tissue injury and inflammation. Increased oxidative stress might lead to the downregulating of serum PON1 activity in pediatric TB patients.

\section{References}

1 Sharda B: Free radicals: emerging challenge in environmental health research in childhood and neonatal disorders. Int $\mathrm{J}$ Environ Res Public Health 2006;3:286-291.

2 Dundaroz R, Erenberk U, Turel O, et al: Oxidative and antioxidative status of children with acute bronchiolitis. J Pediatr 2013;89: 407-411.

3 Cakmak A, Zeyrek D, Atas A, et al: Oxidative status and paraoxonase activity in children with asthma. Clin Invest Med 2009;32:E327E334.

4 Ercan S, Cakmak A, Kösecik M, et al: The oxidative state of children with cyanotic and acyanotic heart disease. Anadolu Kardiyol Derg 2009;9:486-490.

5 Yildiz A, Gur M, Demirbağ R, et al: Paraoxonase and arylesterase activities in untreated dipper and non-dipper hypertensive patients. Clin Biochem 2008;41:779-784.

6 Cooper AM: Cell-mediated immune responses in tuberculosis. Annu Rev Immunol 2009; 27:393-422.

>7 Kwiatkowska S, Szkudlarek U, Luczynska M, et al: Elevated exhalation of hydrogen peroxide and circulating IL-18 in patients with pulmonary tuberculosis. Respir Med 2007;101: 574-580.

8 Betteridge DJ: What is oxidative stress? Metabolism 2000;49:3-8.
-9 Walubo A, Smith PJ, Folb PI: Oxidative stress during antituberculosis therapy in young and elderly patients. Biomed Environ Sci 1995;8: 106-113.

10 Chan J, Tanaka K, Carroll D, et al: Effects of nitric oxide synthase inhibitors on murine infection with Mycobacterium tuberculosis. Infect Immun 1995;63:736-740.

11 Gwinn MR, Vallyathan V: Respiratory burst: role in signal transduction in alveolar macrophages. J Toxicol Environ Health 2006;9:2739.

12 Mackness MI, Mackness B, Durrigton PN: Paraoxonase and coronary heart disease. Atherosclerosis 2002;3:49-55.

13 Aviram M, Rosenblat M: Paraoxonases 1,2 and 3 , oxidative stress, and macrophage foam cell formation during atherosclerosis development. Free Radic Biol Med 2004;37:1304-1316.

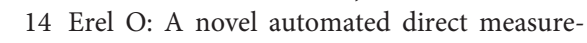
ment method for total antioxidant capacity using a new generation more stable ABTS radical cation. Clin Biochem 2004;37:277-285.

15 Erel O: A new automated colorimetric method for measuring total oxidant status. Clin Biochem 2005;38:1103-1111.

16 Eckerson HW, Wyte MC, La Du BN: The human serum paraoxonase/arylesterase polymorphism. Am J Hum Genet 1083;35:11261138.
17 Nathan C, Shiloh MU: Reactive oxygen and nitrogen intermediates in the relationship between mammalian hosts and microbial pathogens. Proc Natl Acad Sci USA 2000;97: 8841-8848.

18 Dalvi SM, Patil VW, Ramraje NN, et al: Nitric oxide, carbonyl protein, lipid peroxidation and correlation between antioxidant vitamins in different categories of pulmonary and extrapulmonary tuberculosis. Malays J Med Sci 2013;20:21-30

19 Madebo T, Lindtjorn B, Aukrust P, et al: Circulating antioxidants and lipid peroxidation products in untreated tuberculosis patients in Ethiopia. Am J Clin Nutr 2003:78:117-122.

20 Reddy YN, Murthy SV, Krisha DR, et al: Role of free radicals and antioxidants in tuberculosis patients. Indian J Tuberc 2004:51: 213-218.

21 Plit ML, Theron AJ, Fickl H, et al: Influence of antimicrobial chemotherapy and smoking status on plasma concentrations of vitamin C, vitamin $\mathrm{E}$, beta-carotene, acute phase reactants, iron and lipid peroxides in patients with pulmonary tuberculosis. Int J Tuberc Lung Dis 1998;2:590-596.

22 Wiid I, Seaman T, Hoal EG, et al: Total antioxidant levels are low during active $\mathrm{TB}$ and rise with anti-tuberculosis therapy. IUBMB Life 2004;56:101-106. 
-23 Naderi M, Hashemi M, Kpmijani-Bozchaloei F, et al: Serum paraoxonase and arylesterase activities in patients with pulmonary tuberculosis. Pathophysiology 2011;18:117-120.

24 Watson AD, Berliner JA, Hama SY, et al: Protective effect of high density lipoprotein associated paraoxonase. Inhibition of the biological activity of minimally oxidized low density lipoprotein. J Clin Invest 1995;96: 2882-2891.
25 Aviram M, Rosenblat M, Billecke S, et al: $\mathrm{Hu}$ man serum paraoxonase (PON1) is inactivated by oxidized low density lipoprotein and preserved by antioxidants. Free Radic Biol Med 1999;26:794-803.

26 Wickremasingle MI, Thomas LH, Friedland JS: Pulmonary epithelial cells are a source of IL-8 in the response to Mycobacterium tuberculosis: essential role of IL-1 from infected monocytes in a NF- $\kappa B$-dependent network. J Immunol 1999;163:3936-3947.

27 Kumon Y, Nakauchi Y, Suehiro T, et al: Proinflammatory cytokines but not acute phase serum amyloid $\mathrm{A}$ or $\mathrm{C}$ - reactive protein, downregulate paraoxonase 1 (PON1) expression by HepG2 cells. Amyloid 2002;9:160-164.
28 Feingold KR, Memon RA, Moser AH, et al: Paraoxonase activity in the serum and hepatic mRNA levels decrease during the acute phase response. Atherosclerosis 1998;139: 307-315.

29 Selek S, Cosar N, Kocyiğit A, et al: PON1 activity and total oxidant status in patients with active pulmonary tuberculosis. Clin Biochem 2008;41:140-144. 